FM 4:

Planetary nebulae as probes of galactic structure and evolution 


\section{Planetary nebulae as probes of galactic structure and evolution}

\section{Preface}

Focus Meeting 4 (FM4) on Planetary nebulae as probes of galactic structure and evolution was held as part of the XXIXth IAU General Assembly, Honolulu, Hawaii, USA, in August 2015. We had 11 invited reviews, 21 contributed talks, and $\sim 30$ posters. Because of page limitations, we have decided to publish only the invited reviews. In what follows, we attempt to summarize what the reader can expect to find in these proceedings.

Perhaps surprisingly, we have discovered more planetary nebulae $(\mathrm{PNe})$ in other galaxies than we have found in our own. What are the reasons for this? Historically, the first motivation was related to their potential for distance determination. The PN luminosity function (PNLF) was empirically shown to have a constant bright end, which allows it to be used as a standard candle. Twenty-six years ago, the PNLF distances were among the first to point to a Hubble constant near $70 \mathrm{~km} / \mathrm{s} / \mathrm{Mpc}$, subsequently confirmed with other techniques.

We are still trying to understand why the PNLF works so well as a distance indicator. This will eventually lead us to new knowledge of single- and binary-star evolution. The unresolved issues concerning the PNLF were discussed by Robin Ciardullo, and Jason Nordhaus presented an introduction to the physics of the common-envelope phase in close binary evolution, which may play a role in solving the PNLF enigma.

$\mathrm{PNe}$ can be used as probes of the old stellar population in our galaxy and beyond, their chemical abundances are relatively straightforward to calculate, and they represent a definite stage of stellar evolution, thus can be associated to a specific progenitor mass range. The populations of Galactic and Magellanic Cloud PNe have been studied in detail by spectroscopic means to determine the metal distribution in our galaxy and in the Magellanic neighbors. Moving now to harder observational goals, we have started measuring chemical abundances in more distant extragalactic PNe. What limits can we impose on galaxy formation by merging from the observed metallicity gradients in galaxies with a variety of morphologies? This kind of project will eventually need the light-collecting power of 30-m telescopes, but it is time to discuss preliminary strategies, models, and observations.

Paolo Ventura described the challenges of AGB stellar wind modeling with dust, and why it is necessary to carefully test the new model results against PN observations in different populations. Then Anibal García-Hernández showed recent results about dust and molecular content both in our Galaxy (bulge, disk) and in the Magellanic Clouds with their lower metallicity. Next, we had a variety of talks focusing on PN populations in different nearby galaxies. Warren Reid described the PN population of the Large Magellanic Cloud; Bruce Balick reviewed the unexpectedly metal-rich PNe in the very extended halo of M 31; Denise Gonçalves and Michael Richer talked about PN abundances in dwarf galaxies and local spiral galaxies, respectively. Claudia Maraston discussed advanced population synthesis that includes contributions from AGB and postAGB stars. 
It was clear from the very beginning of extragalactic $\mathrm{PN}$ research that $\mathrm{PNe}$ would be excellent kinematic probes of the stellar populations they represent. As soon as new, more efficient slitless methods for radial velocity measurement were implemented, this potential began to be exploited. Lodovico Coccato reviewed our current understanding of PNe as kinematic tracers of galaxy halos. Finally, Magda Arnaboldi described the use of the farthest detectable PNe to study extended galaxy halos and intracluster light in nearby clusters of galaxies.

We had a very stimulating Focus Meeting in Honolulu. We would like to thank the IAU for accepting the FM4 proposal, and all the numerous people who collaborated with an excellent local organization.

Letizia Stanghellini, Miriam Peña, and Roberto Méndez 